\title{
PERSPECTIVAS TERAPÊUTICAS EM METÁSTASES HEPÁTICAS A NEOPLASIA DE COLÓN E RETO
}

\section{ARTIGO DE REVISÃO}

SILVA, Antonia Rafaelly Fernandes ${ }^{1}$

MOREIRA, Geterson Bezerra ${ }^{2}$

CARVALHO, Arthur de Sousa Lima ${ }^{3}$

TELES, João Guilherme Ourique ${ }^{4}$

LOPES, Juan Lucas Furtado ${ }^{5}$

TEXEIRA, Pedro Henrique Machado ${ }^{6}$

VIRGULINO, Stephannie Glozan ${ }^{7}$

COSTA, Thayna Peres ${ }^{8}$

${ }_{1}^{1}$ Discente do curso de Medicina do Centro Universitário UNINTA, Sobral - CE.

${ }^{2}$ Orientador. Cirurgião Geral. Médico Endoscopista. Coordenador da Residência em Cirurgia, Sobral - Ceará.

${ }^{3}$ Discente do curso de Medicina Faculdade Santa Maria - FSM.

4 Discente do curso de Medicina do Centro Universitário UNINTA, Sobral - CE.

${ }^{5}$ Discente do curso de Medicina do Centro Universitário INTA- UNINTA, Sobral - CE.

${ }^{6}$ Discente do curso de Medicina da Faculdade de Medicina de Itajubá - FMITCampus Itajubá.

7 Discente do curso de Medicina do da Universidade de São Paulo - UNICID Campus São Paulo.

${ }^{8}$ Discente do curso de Medicina da Faculdade de Ciências Humanas, Exatas e da Saúde do Piauí - Parnaíba. 
ARAÚJO, Yasmim Tapety ${ }^{9}$

VIANA, Ana Luíza Soares ${ }^{10}$

SILVA, Antonia Rafaelly Fernandes. Et al. Perspectivas terapêuticas em metástases hepáticas a neoplasia de colón e reto. Revista Científica Multidisciplinar Núcleo do Conhecimento. Ano 05, Ed. 10, Vol. 05, pp. 93-110. Outubro de

2020.

ISSN:

2448-0959,

Link

de

acesso: https://www.nucleodoconhecimento.com.br/saude/perspectivas-terapeuticas

\section{RESUMO}

Introdução: O câncer colorretal é um dos cânceres mais importantes e recorrentes do Brasil. Associado a estes eventos estão, comumente, quadros metastáticos, preferencialmente as lesões hepáticas, sendo assim, este estudo objetiva identificar as terapêuticas de maior ou menor morbimortalidade deste evento. Metodologia: foi realizada uma busca dos descritores relacionados à temática, os quais foram identificados utilizando o DeCS (Descritores em Ciência da Saúde). Os descritores oportunos foram sendo estes: "Metastases", "Carcinoma Colorectal", "Hepatectomia", "Morbidades", "mortalidade", "Estudo Comparativo", "ressecção de metástases hepáticas", "complicações pós-operatória estes foram ajustados em formula de buscas aplicadas no BVS e PubMed, alcançando um número de 113 artigos (88 na primeira e 25 na segunda). Após seguidos processos de inclusão e exclusão, foram pré-definidos 10 artigos originais restaram para serem revisados. Resultados: Sobre os fatores intrínsecos ao paciente, constatou-se a necessidade de conduta individualizada, em que idade e tipo histológico são fatores preponderantes. As vias cirúrgicas mostraram melhores desfechos por via laparoscópica, em metástases solitárias, além de abordagens anatômicas, evidenciando também vantagens a técnica da cirurgia robótica, porém de difícil acesso. Discussão e Considerações finais: O presente trabalho propôs uma revisão acerca do verdadeiro benefício da

${ }^{9}$ Discente do curso de Medicina do Centro Universitário UNINTA, Sobral - CE. 10 Discente do curso de Medicina do Centro Universitário - UNIFACID, Teresina- PI. 
hepatectomia em pacientes com metástase hepática por câncer colorretal e suas possíveis complicações, a fim de estabelecermos os melhores meios para a sobrevida desses pacientes.

Palavras Chave: Metástases Hepáticas, hepatectomia, morbidade, mortalidade.

\section{INTRODUÇÃO}

Um dos cânceres mais incidentes e importantes no Brasil é o chamado câncer colorretal, esse grupo abrange os tumores que aparecem desde a porção do intestino grosso chamado de cólon até o reto e ânus. Somente no ano de 2020 o Instituto Nacional do Câncer (INCA) apurou mais de 40 mil novos casos, dessa população oncológica e se estima que cerca de $23 \%$ já se encontra com alguma metástase hepática isolada. O que evidencia a importância do tema visto que a cura desses pacientes pode ser alcançada com uma rápida intervenção cirúrgica a qual a sobrevida média é de mais de 30\% em 5 anos de operação. (INCA, 2020)

O fator de risco isolado mais considerado para o aparecimento desse câncer é a idade, tanto que, a diretriz brasileira, feita por várias sociedades médicas, preconiza que seja feito o rastreio do sangue oculto nas fezes anualmente a partir do 50 anos de idade somada a uma retossigmoidoscopia a cada 5 anos em indivíduos de baixo risco. $\mathrm{O}$ rastreio possui uma função fundamental nessa neoplasia, pois o diagnóstico rápido com a intervenção cirúrgica adequada muda o prognóstico do doente de forma positiva, principalmente nas metástases hepáticas que representam $2 / 3$ dos óbitos da doença tumoral. (FANG CHIA, 2002)

Infelizmente, apesar do notório benefício, estima-se que apenas $20 \%$ a $25 \%$ das metástases hepáticas são potencialmente operáveis no momento do seu diagnóstico, deixando a ressecção cirúrgica apenas em situações restritas. Para um paciente ser considerado portador de doença neoplásica ressecável, deve-se garantir a possibilidade de exérese total da lesão (macro e microscópico) com vascularização completa, drenagem biliar efetiva e um volume hepático satisfatório, além disso, margens livre de pelo menos $1 \mathrm{~cm}$ são recomendadas na ressecção tumoral. Vale 
ressaltar que a presença ou não de doença tumoral extra-hepática e ressecável não contraindica o tratamento cirúrgico das lesões hepáticas. (LUPINACCI, 2013)

O adenocarcinoma é o tipo histológico mais comum de câncer colorretal que faz metástase hepática, a cirurgia dessas metástases de chama hepatectomia e pode ser feita por via laparoscópica (videoscópia) ou por laparotomia (aberta). O procedimento cirúrgico é dividido em anatômicos e não anatômicos, o prognóstico do paciente é o mesmo nos dois tipos de intervenções, desde que as margens histológicas negativas sejam alcançadas com sucesso.

Nos países ocidentais a técnica mais utilizada é a hemi-hepatectomia, pelo seu potencial de preservação da função hepática no pós-cirúrgico, conferindo ao doente uma tolerância maior ao procedimento e uma facilidade técnica ao cirurgião. Em pacientes considerados com tumores não ressecáveis, opta por meios de aumentar o potencial de ressecção da neoplasia. Isso é comumente chamado de hepatectomia de dois estágios, onde, na primeira etapa se faz a embolia da vascularização do carcinoma a fim de reduzir a área tumoral no fígado, dessa forma, deixando a lesão longe de estruturas nobres e facilitando sua ressecção que é a segunda etapa do procedimento. (AKGÜL et al., 2014)

Diante do exposto, fica clara a importância da hepatectomia das metástases para a sobrevivência dos pacientes vítimas do câncer colorretal, aumentando o prognóstico e a qualidade de vida dos enfermos. Esse estudo tem como objetivo analisar esses resultados pós-cirurgia, estudando a relação entre o real benefício cirúrgico e a morbidade que o procedimento possui.

\section{DESENVOLVIMENTO}

\subsection{METODOLOGIA}

Trata-se de uma revisão bibliográfica integrativa do tipo qualitativa, com intuito descritivo de estudos nacionais e internacionais. Outrossim, a natureza dessa pesquisa enquadra-se como uma pesquisa básica e abrangente de coleta de dados 
em estudos originais, apresentação de resultados e confrontação com a literatura atual, segundo os autores.

A princípio, foi realizada uma busca dos descritores relacionados à temática, os quais foram identificados utilizando o DeCS (Descritores em Ciência da Saúde). Os descritores oportunos foram sendo estes: "Metastases", "Carcinoma Colorectal", "Hepatectomia", "Morbidades", "mortalidade", "Estudo Comparativo", "ressecção de metástases hepáticas", "complicações pós-operatória", possibilitando também a associação desses termos em inglês e espanhol na fórmula de busca.

A pesquisa foi realizada por meio das bases de dados online do Portal da Biblioteca Virtual em Saúde (BVS) como também no PubMed, possuindo as seguintes fórmulas de busca (tw:(Metastic Colorectal cancer )) OR (tw:(Colorectal liver metastasis )) AND (tw:(Resection of colorectal liver metastasis )) OR (tw:(Hepatectomy )) AND (tw:(Intraoperative complication )) OR (tw:(postoperative complication )) AND (tw:(Morbidity)) OR (tw:(Mortality)) AND (tw:(outcome)), além desta Neoplasm Metastases OR Metastasis OR Metastases OR Metastasis, Neoplasm and Liver Neoplasms OR Tumors OR hepatic involvement AND NOT primary liver tumor AND Colorectal Neoplasm OR Colorectal Carcinoma OR Colorectal Tumors OR Metastatic colorectal cancer resection of colorectal liver metastasis or hepathectomy OR hepatectomies AND Surgical injury OR Surgical injuries OR Intraoperative Complication AND morbidities OR morbidity AND mortalities OR Case Fatality rate OR Case Fatality Rates OR Excess Mortalities Comparative Studies OR Comparative Study AND Clinical Evolution Outcome Assessment OR Health Care utilizada no PubMed. Essas fórmulas foram aplicadas as bases de dados supracitadas, BVS e PubMed, respectivamente, alcançando um número de 113 artigos (88 na primeira e 25 na segunda).

Inicialmente, precedeu-se uma busca de palavras-chave pertinentes a questão norteadora do presente estudo, que é a morbimortalidade das metástases hepáticas em tumores de cólon e reto e o impacto cirúrgico no desfecho clínico destes quadros. Após a busca dos artigos compreendidos nos critérios preestabelecidos, a primeira etapa de seleção compreendeu a exclusão de artigos repetidos, seguida por uma 
análise de título e por último a leitura dos resumos. Nesta primeira etapa, foram selecionados 38 artigos ( 31 artigos na plataforma BVS e 7 artigos no PubMed).

Neste momento, após leitura integral dos 38 títulos, os artigos foram analisados segundo critérios de inclusão e exclusão previamente elencados, sendo estes os critérios de inclusão: estudos publicados a partir de 2015; artigos que estudassem somente o evento principal da pesquisa, ou seja, intervenções cirúrgicas no sítio hepático e artigos originais. Os critérios de exclusão utilizados foram artigos que expusessem :pacientes com mais de 80 anos; envolvimento de outros órgãos ao procedimento cirúrgico e artigos com leitura indisponível.

Finalmente, foram eleitos 10 artigos os quais serão estudados e analisados para apresentação deste estudo, segundo os seguintes parâmetros, os quais serão demonstrados em tabela: Título, autor, ano, tipo de estudo, número de participantes do estudo, intervenção terapêutica, desfecho clínico e resultados.

Vale salientar que a pesquisa não apresenta caráter prático, o que dispensa, portanto, a necessidade de submissão ao Comitê de Ética em Pesquisa (CEP).

\subsection{RESULTADOS}

Tabela 1 - Quadro 01: Tabela de resultados.

\begin{tabular}{|c|c|c|c|}
\hline Autor & $\begin{array}{l}\text { Tipo de } \\
\text { estudo }\end{array}$ & Intervenção & Resultados \\
\hline $\begin{array}{l}\text { NACHMANY, } \\
\text { Ido et al., } \\
2016 \text {. }\end{array}$ & $\begin{array}{l}\text { Caso- } \\
\text { controle }\end{array}$ & $\begin{array}{lr}\text { Ressecção } & \text { de } \\
\text { metástases } & \text { hepáticas } \\
\text { colorretais } & \text { em } \\
\text { pacientes } & \text { idosos,em } \\
\text { torno de } 70 \text { anos, que } \\
\text { estivessem } \\
\text { cuidados paliativos } \\
\text { num período de } 5 \text { anos }\end{array}$ & $\begin{array}{l}\text { A intervenção cirúrgica } \\
\text { demonstrou impacto } \\
\text { positivo na sobrevida e } \\
\text { qualidade de vida, } \\
\text { apresentando } 7 \text { a } 20 \% \text { de } \\
\text { índices curativos nas } \\
\text { ressecções }\end{array}$ \\
\hline
\end{tabular}




\begin{tabular}{|c|c|c|c|}
\hline $\begin{array}{l}\text { GAGNIÈRE, } \\
\text { Johan et al., } \\
2020\end{array}$ & Coorte & $\begin{array}{l}\text { Análise do impacto das } \\
\text { ressecções hepáticas } \\
\text { em pacientes com } \\
\text { câncer colorretal } \\
\text { mutante BRAF }\end{array}$ & $\begin{array}{l}\text { Os pacientes com } \\
\text { mutante BRAF eram } \\
\text { comumente mais velhos, } \\
\text { com maiores pontuações } \\
\text { ASA. Resultados } \\
\text { mostram que embora } \\
\text { operáveis, apresentam } \\
\text { altas taxas de } \\
\text { recorrência do quadro }\end{array}$ \\
\hline $\begin{array}{l}\text { GURUSAMY, } \\
\text { Kurinchi et al., } \\
2018\end{array}$ & $\begin{array}{l}\text { Ensaio clínico } \\
\text { randomizado }\end{array}$ & $\begin{array}{l}\text { Uso da ablação térmica } \\
\text { em detrimento da } \\
\text { ressecção cirúrgica nas } \\
\text { metástases hepáticas } \\
\text { de tumores colorretais } \\
\text { em pacientes com } \\
\text { metástases curáveis } \\
\text { sem outras } \\
\text { malignidades } \\
\text { concomitantes }\end{array}$ & $\begin{array}{l}\text { Houve uma sobrevida } \\
\text { livre de doenças após } \\
\text { dois anos da } \\
\text { randomização, não } \\
\text { demonstrando } \\
\text { inferioridade da ablação } \\
\text { nos pacientes eleitos } \\
\text { candidatos. }\end{array}$ \\
\hline $\begin{array}{l}\text { VANDER } \\
\text { POEL, M. J. } \\
\text { et al., } 2019 .\end{array}$ & $\begin{array}{l}\text { Caso } \\
\text { controle }\end{array}$ & $\begin{array}{l}\text { Análise do desfecho } \\
\text { clínico entre pacientes } \\
\text { com ressecção } \\
\text { laparoscópica } \\
\text { combinada e a } \\
\text { ressecção hepática } \\
\text { isolada, por meio de } \\
\text { confrontação de casos } \\
\text { por testes pareados } \\
\text { num período de sete } \\
\text { anos }\end{array}$ & 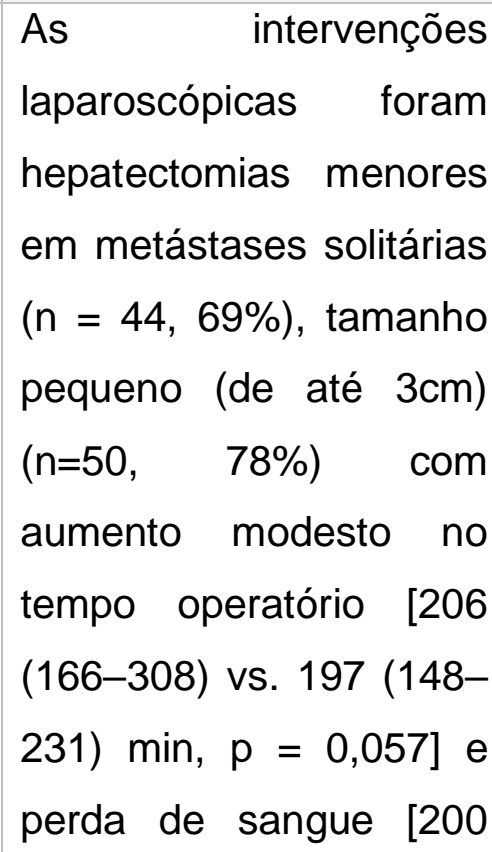 \\
\hline
\end{tabular}




\begin{tabular}{|c|c|c|c|}
\hline & & & $\begin{array}{l}\text { (100-700) vs. } 75 \text { (5-200) } \\
\text { ml, p = 0,011. A } \\
\text { mortalidade em } 30 \text { dias } \\
\text { após a operação foram } \\
\text { semelhantes nas duas } \\
\text { intervenções. }\end{array}$ \\
\hline $\begin{array}{l}\text { UBINK, Inge } \\
\text { et al., } 2016 .\end{array}$ & $\begin{array}{l}\text { Estudo } \\
\text { retrospectivo }\end{array}$ & $\begin{array}{l}\text { Análise retrospectiva } \\
\text { de desfechos } \\
\text { oncológicos } \\
\text { cirúrgicos em pacientes } \\
\text { submetidos a cirurgias } \\
\text { de grande porte para } \\
\text { metástases hepáticas } \\
\text { de tumor colorretal num } \\
\text { hospital universitário } \\
\text { holandês no período de } \\
2000 \text { a } 2015\end{array}$ & 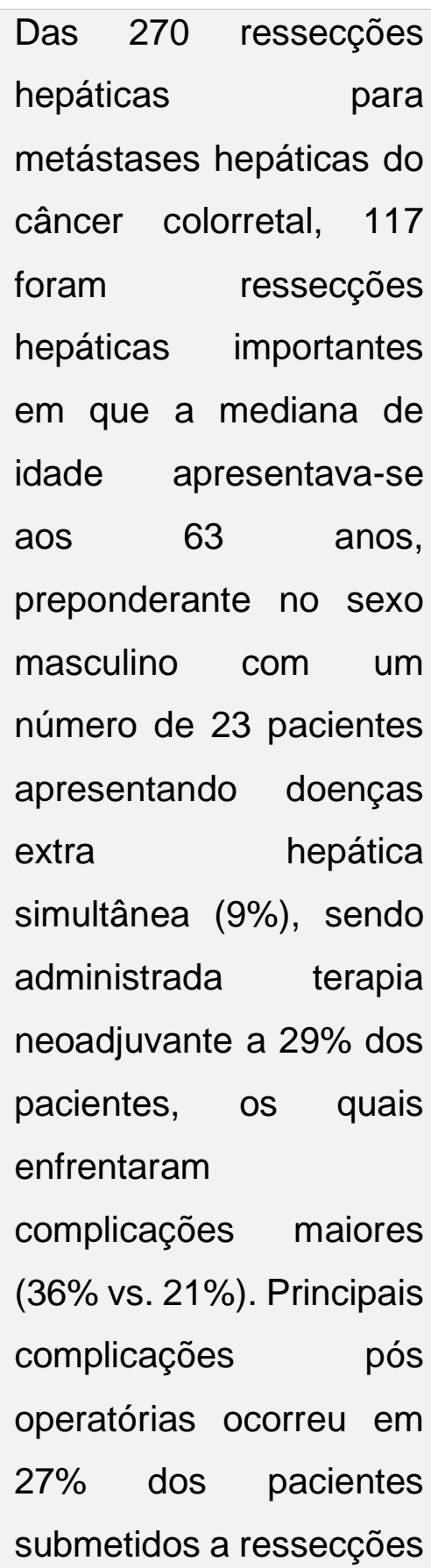 \\
\hline
\end{tabular}




\begin{tabular}{|c|c|c|c|}
\hline & & & $\begin{array}{l}\text { maiores. As causas de } \\
\text { morte após ressecção } \\
\text { importante foram as } \\
\text { seguintes: insuficiência } \\
\text { hepática (n } 1 / 42 \text { ), falha de } \\
\text { múltiplos órgãos (n } 1 / 42 \text { ), } \\
\text { pancreatite ( } \mathrm{n} \text { T1/4 1), } \\
\text { aguda síndrome da } \\
\text { dificuldade respiratória ( } \mathrm{n} \\
\text { 1/4 2), embolia pulmonar } \\
\text { (n 1/4 } 1 \text { ) e hemorragia } \\
\text { intra-abdominal (n = } 1 \text { ). } \\
\text { Recorrência e sistema } \\
\text { operacional } \\
\text { acompanhamento médio } \\
\text { após Hepatectomia } \\
\text { importante foi de } 22,5 \\
\text { meses. }\end{array}$ \\
\hline $\begin{array}{l}\text { PESI, } \\
\text { Benedetta et } \\
\text { al, } 2019 \text {. }\end{array}$ & $\begin{array}{l}\text { Estudo } \\
\text { prospectivo }\end{array}$ & $\begin{array}{l}\text { Análise de todos os } \\
\text { pacientes que } \\
\text { receberam ressecção } \\
\text { robótica para tumores } \\
\text { malignos hepáticos }\end{array}$ & $\begin{array}{l}\text { A análise foi realizada } \\
\text { durante o período de } \\
\text { setembro de } 2008 \text { a } \\
\text { janeiro de } 2017 \text {, } \\
\text { possuindo um perfil } \\
\text { epidemiológico de } 24 \\
\text { mulheres e } 27 \text { homens } \\
\text { com a média de idade de } \\
64 \quad \text { anos. Destes } \\
\text { pacientes } 13 \text { foram } \\
\text { submetidos } \\
\text { hemihepatectomia e } 38 \\
\text { a cirurgia de grande }\end{array}$ \\
\hline
\end{tabular}




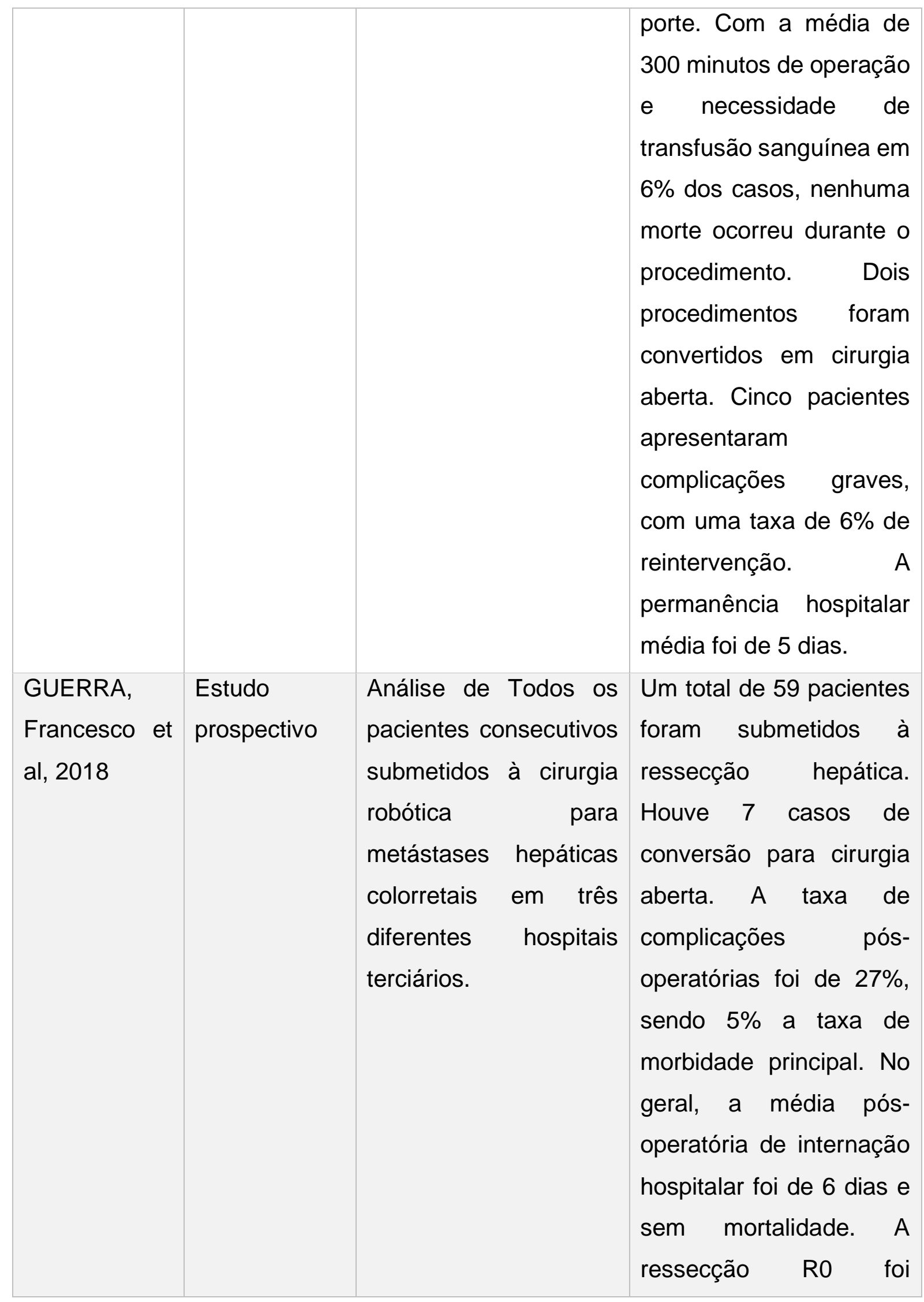




\begin{tabular}{|c|c|c|c|}
\hline & & & $\begin{array}{l}\text { alcançada em } 92 \% \text { das } \\
\text { lesões. } \\
\text { acompanhamento dos } \\
\text { pacientes foi numa média } \\
\text { de } 19 \text { meses, o DFS de } 1 \\
\text { ano e } 3 \text { anos foi de } 83,5 \% \\
\text { e } 41,9 \% \text {, enquanto o OS } \\
\text { de } 1 \text { e } 3 \text { anos foi } 90,4 \% \text { e } \\
66,1 \% \text {, respectivamente }\end{array}$ \\
\hline $\begin{array}{l}\text { SHE, Wong et } \\
\text { al, } 2020 \text {. }\end{array}$ & $\begin{array}{l}\text { Estudo } \\
\text { retrospectivo. }\end{array}$ & $\begin{array}{l}\text { Análise e confrontação } \\
\text { de todos os pacientes } \\
\text { submetidos a com } \\
\text { hepatectomia anatômica } \\
\text { ressecção de } \\
\text { ou não anatômica de } \\
\text { janeiro de } 1990 \text { a } \\
\text { janeiro de } 2017\end{array}$ & 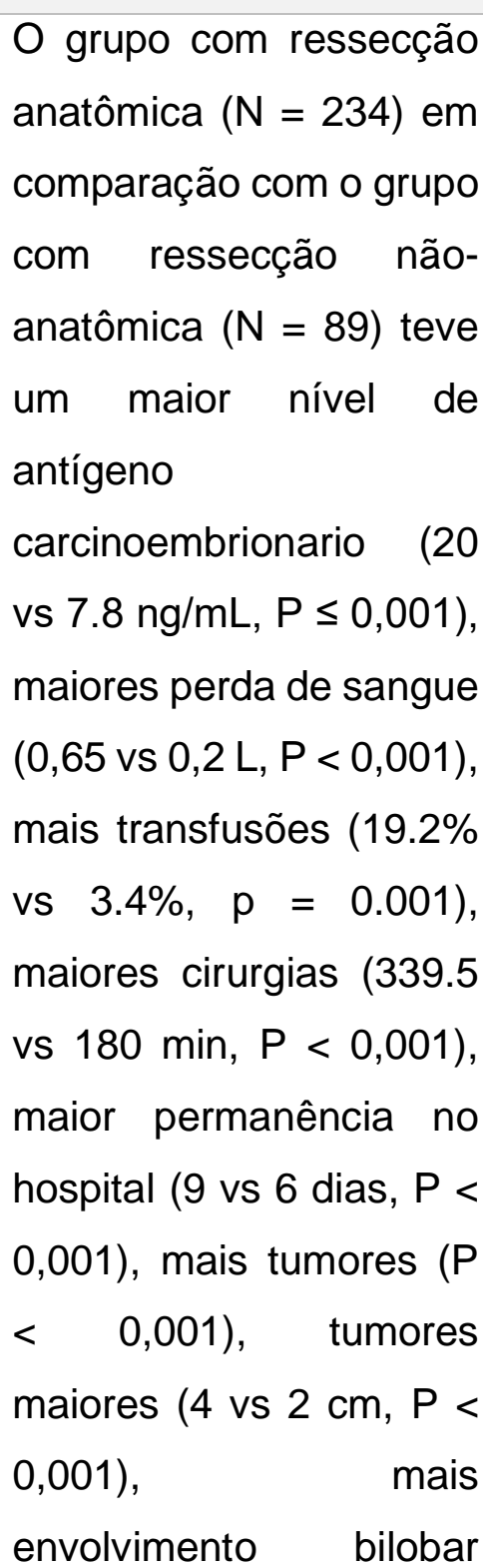 \\
\hline
\end{tabular}




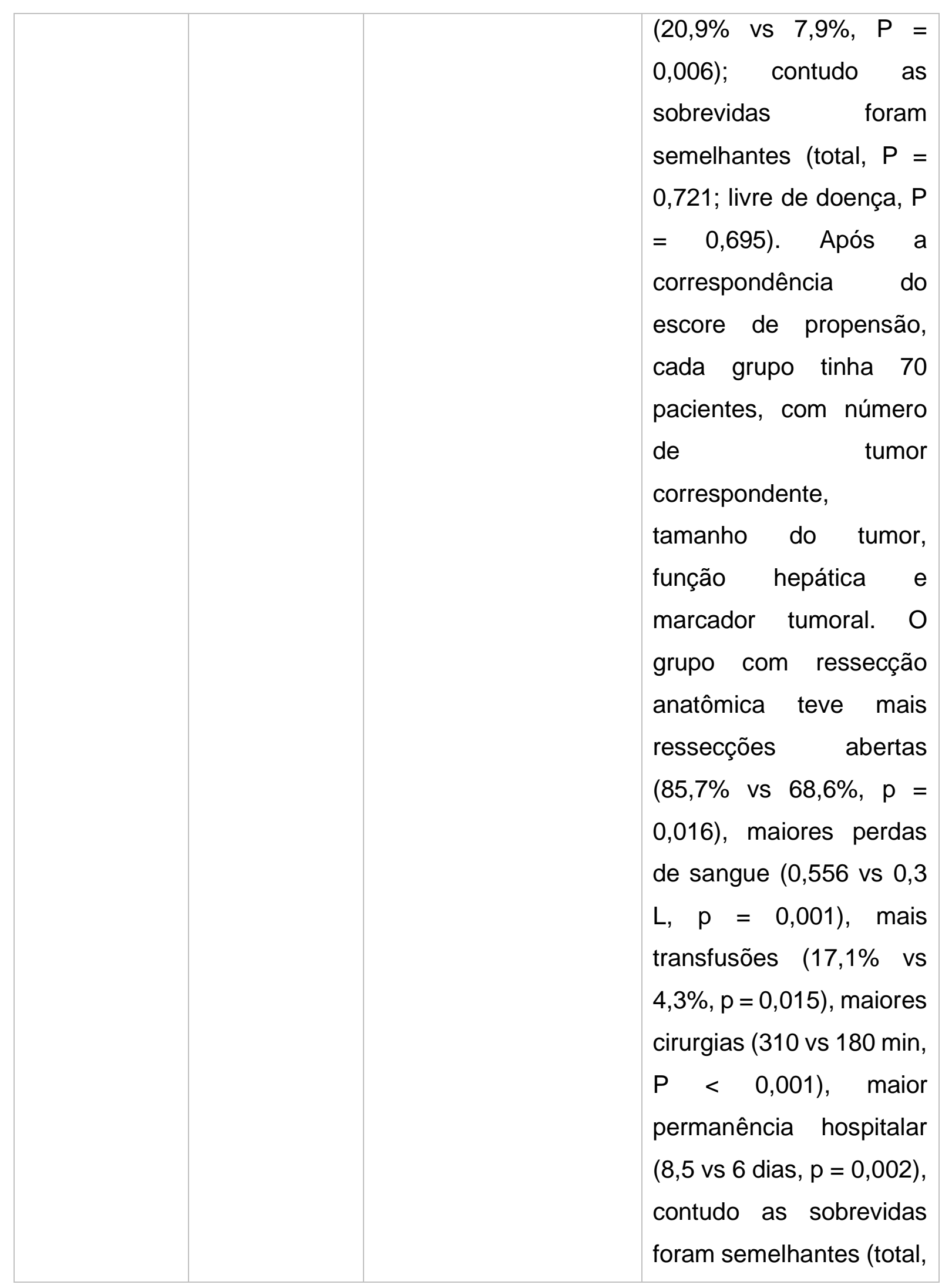




\begin{tabular}{|c|c|c|c|}
\hline & & & $\begin{array}{l}P=0,819 ; \text { livre de } \\
\text { doença, } P=0,855) .\end{array}$ \\
\hline $\begin{array}{l}\text { DEDINSKÁ, } \\
\text { Ivana et al, } \\
2020 \text {. }\end{array}$ & $\begin{array}{l}\text { Estudo } \\
\text { retrospectivo. }\end{array}$ & $\begin{array}{l}\text { Análise retrospectiva } \\
\text { Todos os pacientes } \\
\text { foram submetidos a } \\
\text { ressecção hepática de } \\
2014 \text { a } 2013 \text { através da } \\
\text { identificação do tipo } \\
\text { histológica e técnica } \\
\text { empregada (grande } \\
\text { (hemihepatectomia ou } \\
\text { hemihepatectomia } \\
\text { estendida), pequeno } \\
\text { (ressecção de } \\
\text { segmentos), ou } \\
\text { ablação } \\
\text { radiofrequência } \\
\text { técnica foi realizada } \\
\text { quando o achado no } \\
\text { fígado não pôde ser } \\
\text { ressecado) }\end{array}$ & 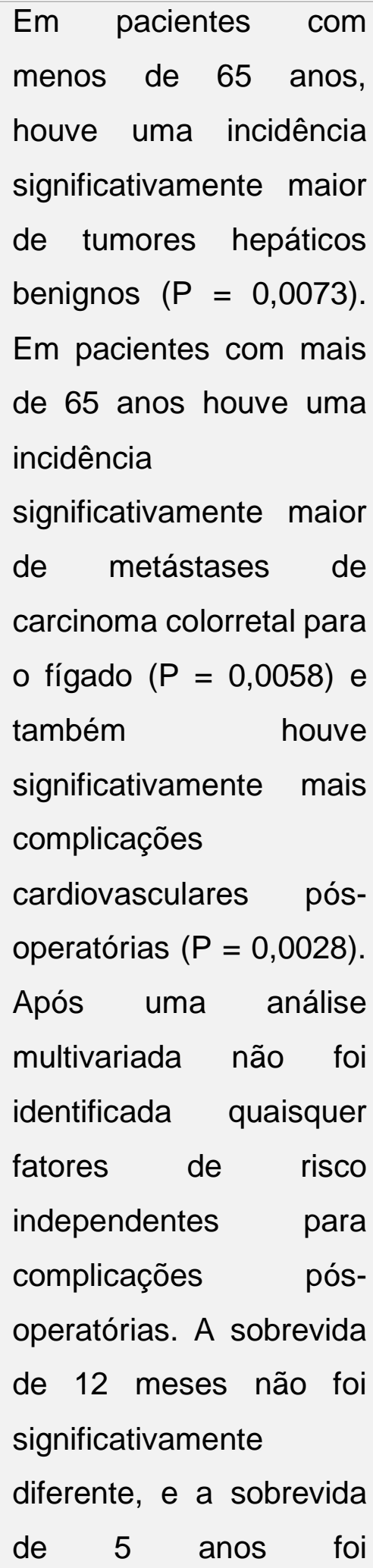 \\
\hline
\end{tabular}




\begin{tabular}{|c|c|c|c|}
\hline & & & $\begin{array}{l}\text { significativamente pior } \\
\text { em pacientes mais } \\
\text { velhos }(P=0,0454) \text {. }\end{array}$ \\
\hline $\begin{array}{l}\text { FUKAMI, } \\
\text { Yasuyuki et } \\
\text { al, } 2016 .\end{array}$ & $\begin{array}{l}\text { Estudo } \\
\text { retrospectivo, } \\
\text { não } \\
\text { randomizado. }\end{array}$ & $\begin{array}{l}\text { Análise retrospectiva } \\
\text { dos pacientes foram } \\
\text { submetidos à } \\
\text { hepatectomia curativa } \\
\text { primária por metástase } \\
\text { de câncer colorretal. }\end{array}$ & $\begin{array}{l}\text { Doa } \\
\text { analisados, } \\
\text { pacientes } 193,39 \%) \\
\text { desenvolveram } \\
\text { recorrência, e ama } \\
\text { hepatectomia foi repetida } \\
\text { em } 62 \text { pacientes. Dos } 62 \\
\text { paciente reoperados pela } \\
\text { segunda vez, 11 foram } \\
\text { pela terceira vez, } 4 \text { pela } \\
\text { quarta vez, } 1 \text { pela quinta } \\
\text { vez. As taxas de } \\
\text { morbidade e mortalidade } \\
\text { pós-operatórias foram } \\
\text { baixas (11,5 e } 1,3 \% \text {, } \\
\text { respectivamente). As } \\
\text { taxas de sobrevida global } \\
\text { foram de } 3 \text { e } 5 \text { anos após } \\
\text { hepatectomia primária } \\
\text { para metástases de } \\
\text { câncer colorretal na } \\
\text { hepatectomia } \\
\text { repetição foram } 79,5 \text { e } \\
57,4 \%, \text { respectivamente. }\end{array}$ \\
\hline
\end{tabular}

Fonte: Próprio autor.

Foram analisados 10 estudos que tinham como característica primordial serem estudos originais, dos quais $40 \%$ correspondem a estudos retrospectivos $(n=4)$, sendo 
esta a maior fração deste estudo, $20 \%$ de estudos prospectivos $(n=2), 20 \%$ de estudos do tipo caso controle $(n=2), 10 \%$ fora representado por ensaio do tipo coorte $(n=1)$, e $10 \%$ do tipo ensaio clínico randomizado. Com relação ao ano de publicação, como apresentado no Quadro 1, o ano de 2016 concentrou maior número de artigos utilizados, seguindo um em 2018, 2019, 2020.

Destes trabalhos, $40 \%(n=4)$ avaliaram as indicações cirúrgicas conforme as condições inerentes ao paciente e apresentação de sua doença: paciente idoso e/ou em cuidados paliativos, tipo histológico do tumor apresentado e a presença do mutante BRAF. Como também, $40 \%(n=4)$ destes trazem comparações acerca do método utilizado e como estes impactam no desfecho cirúrgico e oncológico (uso de ressecção por cirurgia aberta, laparoscópica ou robótica). Além disso, 20\% ( $n=2)$ dizem respeito a uso de técnicas conservadoras ou agressivas. Desta forma, estabeleceu-se as seguintes categorias para avaliação primária dos resultados:

\subsection{FATORES INERENTES AOS PACIENTES}

Dos quatro estudos analisados neste quesito, 20\% $(n=2)$ dos referidos estudos tratavam sobre a escolha de abordagem cirúrgica em pacientes idosos, um deste relatava quadro conjunto a condição de idosos em cuidados paliativos. O primeiro, um estudo de caso controle realizado revelou impacto positivo na sobrevida destes pacientes, afirmando inclusive ter tido desfecho curativo nestas metástases em 7\% a $20 \%$ dos casos analisados por uma média de tempo de cinco anos (NACHMANY et al., 2016). O segundo, o qual se propunha a pesquisar o pós operatório em pacientes geriátricos, demonstrou baixa morbidade e mortalidade pós cirúrgica, $(11,5 \%$ e 1,3\% respectivamente), no entanto as taxas de necessidade a reabordagem foi de $68,39 \%$ de um total de 193 pacientes em um $n=132$ (DEDINSK et al., 2020).

Quanto ao tipo histológico e presença do BRAF mutante, relatados em dois outros estudos anteriormente apresentados demonstram que a variância do primeiro, como a presença do segundo impactam de maneira vultosa o desfecho clínico e cirúrgico, como também os critérios pré-operatórios como a classificação de estado físico 
ASA (Sociedade Americana de Anestesiologistas) (UBINK et al., 2016) (GAGNIER et al., 2020).

\subsection{VIAS CIRÚRGICAS}

Estes estudos trazem comparações de abordagens abertas, via laparoscópica e conduta robótica. Um dos estudos analisados demonstrou que em metástases solitárias como demonstrada em $69 \%$ dos pacientes abordados $(n=44)$ de tamanho pequeno (de até $3 \mathrm{~cm}$ ) a via laparoscópica pode ser preferida com bons desfechos e configurando uma abordagem menos invasiva (VANDER et al., 2020) Um outro método não amplamente utilizado estudado se deu por meio de ablação térmica não demonstrou inferioridade em detrimento a métodos de ressecções convencionais, desde que estes pacientes não possuíssem outros acometimentos neoplásicos concomitantes (GURUSAMY et al., 2018). A cirurgia robótica, apresentou, segundo estudos, baixas taxas de complicação e morbidade, sendo o tempo de internação para observação pós cirúrgica de cinco a seis dias (GUERRA et al., 2018) (PESI et al., 2019)

\subsection{CIRURGIAS DE GRANDE PORTE E RESSECÇÕES CONSERVADORAS}

Existe resultados acerca da necessidade de reoperação em abordagens conservadoras, como também a indicação de complicações pós cirúrgicas em operações de grande porte. As causas de morte após ressecção importante foram as seguintes: insuficiência hepática ( $n$ 1/4 2), falha de múltiplos órgãos ( $n$ 1/4 2), pancreatite (n T1/4 1), aguda síndrome da dificuldade respiratória (n 1/4 2), embolia pulmonar ( $n$ 1 1/41) e hemorragia intra-abdominal $(n=1)$ (UBINK et al., 2016). No que tange a escolha de ressecções, grupo com ressecção anatômica $(\mathrm{N}=234)$ em comparação com o grupo com ressecção não-anatômica $(N=89)$ teve um maior nível de antígeno carcinoembrionario (20 vs $7.8 \mathrm{ng} / \mathrm{mL}, \mathrm{P} \leq 0,001)$, maiores perda de sangue $(0,65$ vs $0,2 \mathrm{~L}, \mathrm{P}<0,001)$, mais transfusões $(19.2 \%$ vs $3.4 \%, p=0.001)$, maiores cirurgias (339.5 vs 180 min, $P<0,001$ ), maior permanência no hospital (9 vs 6 dias, $P<0,001$ ), 
mais tumores $(P<0,001)$, tumores maiores ( 4 vs $2 \mathrm{~cm}, P<0,001$ ), mais envolvimento bilobar $(20,9 \%$ vs $7,9 \%, P=0,006)$; contudo as sobrevidas foram semelhantes (total, $P=0,721$; livre de doença, $P=0,695)-($ SHE et al., 2020)

\subsection{DISCUSSÃO}

O câncer colorretal tem íntima relação com a idade sendo a sua magnificência em pessoas acima de 50 anos de idade. No entanto confrontando tal aspecto, estudos revelam um decréscimo nos números de casos em idosos se comparado a um aumento exponencial na população mais jovem, podendo tal associação ter correlação com o aumento do rastreio (CAMPOS et al., 2017)

No presente estudo, ainda foi revelado um impacto positivo nas cirurgias oncológicas em paciente geriátricos acrescentando ainda um bom desfecho clínico e uma redução na morbidade e mortalidade cirúrgica. Em contrapartida, estudo randomizado realizado no Japão com 55 pacientes categorizados conforme intervalo de idade (55 a 64 anos, 65 a 75 e maiores que 75), conduzido em centro oncológico, mostrou que a idade cronológica é um relevante fator para o risco de morbidade operatória e mortalidade precoce em cirurgias oncológicas de grande porte, reiterando, portanto, a avaliação da extensão e quantidade de lesões para a abordagem operatória (YOO et al., 2016)

Com relação ao acréscimo negativo evidente do riscos de um desfecho clínico e cirúrgicos desfavoráveis associado ao tipo histológico e a presença do gene de mutação BRAF os estudos consentiram com o enorme impacto no prognósticos do câncer de cólon retal e ressaltou a importância da determinação histológica da profundidade de invasão tumoral e metástases linfonodais (VOLPATO; KOCH, 2018).

O papel benéfico da cirurgia laparoscópica na ressecção do câncer de cólon no artigo houve a ratificação e por fim o estabelecimento de que a cirurgia laparoscópica quando comparada a cirurgia aberta tradicional traz benefícios em curto prazo com menor dor, tempo de internação e retorno antecipado da motilidade intestinal (LEE, 2009). Desta maneira, infere-se a consolidação da via laparoscópica em detrimento 
da abordagem de cirurgia aberta, com poucos números de conversão de uma via para outra.

Houve Consenso quanto ao benefício de abordagem de metástases hepáticas solitárias por via laparoscópica (CHERQUI et al., 2017) (VANDER et al., 2019) em que observou-se evidentes vantagens com respeito a esta via cirúrgica, dentre as quais estavam a menor incidência de ascite e menores complicações pós operatórias. A maior frequência relatada na literatura de hepatectomia laparoscópica foi para doenças benignas ou tumores benignos do fígado.

Embora promissores, a técnica de ablação e cirurgia por via robótica ainda requerem altos custos e treinamentos ainda não difundidos. Em revisão sistemática e metanálise, ressaltou-se as vantagens na abordagem hepática com ampliação tridimensional da imagem e menor risco de erros operatórios pois permite abordar de maneira minimamente invasiva com ampla visão do campo operatório (MONTALTI et al., 2015)

Quanto as ressecções, a presença de tumores no sítio hepático apresentam variados tratamentos conforme a apresentação da lesão. A abordagem cirúrgica pode ser conservadora ou ampliada, bem como a hepatectomia pode ser central ou mesohepatectomia (SHE et al., 2020). Segundo a literatura, esta última apresenta menor morbidade, e a abordagem anatômica superioridade a não anatômica (SCUDAMORE et al., 2000)

\section{CONSIDERAÇÕES FINAIS}

Através dos dados analisados, pode-se inferir um benefício em detrimento do malefício quando realizado a hepatectomia em pacientes metastáticos, pois as evidências mostram que há formas de propiciar melhor sobrevida a estes. Os melhores resultados são influenciados pela idade cronológica, extensão e quantidade de lesões, características histológicas, presença de mutações e o tipo de abordagem, fatores de extrema relevância. Sendo assim, uma abordagem adequada e um rastreamento ideal possibilitam um diagnóstico oportuno e, portanto, uma intervenção 
precoce com melhores prognósticos. Além disso, uma abordagem laparoscópica pode proporcionar melhores condições pós-cirurgia ao paciente, visto que reduz o tempo de internação e suas complicações.

\section{REFERÊNCIAS}

AKGÜL, Özgür et al. Role of surgery in colorectal cancer liver metastases. World Journal Of Gastroenterology, [S.L.], v. 20, n. 20, p. 6113-6120, 2014. Baishideng Publishing Group Inc.. http://dx.doi.org/10.3748/wjg.v20.i20.6113

CAMPOS, Fábio Guilherme C. M. de et al. Incidence of colorectal cancer in young patients. Revista do Colégio Brasileiro de Cirurgiões, [S.L.], v. 44, n. 2, p. 208-215, abr. 2017. FapUNIFESP (SciELO). http://dx.doi.org/10.1590/0100-69912017002004.

DEDINSKÁ, Ivana et al. Complications of Liver Resection in Geriatric Patients. Annals Of Hepatology, [S.L.], v. 16, n. 1, p. 149-156, jan. 2017. Elsevier BV. http://dx.doi.org/10.5604/16652681.1226934.

FANG CHIA, BIN. Rastreamento para câncer colorretal. Rev. Assoc. Med. Bras., São Paulo, v. 48, n. 4, p. 286, dez. 2002. Disponível em: https://doi.org/10.1590/S010442302002000400020

FUKAMI, Yasuyuki et al. Postoperative complications following aggressive repeat hepatectomy for colorectal liver metastasis have adverse oncological outcomes. Surgery Today, [S.L.], v. 47, n. 1, p. 99-107, 27 abr. 2016. Springer Science and Business Media LLC. http://dx.doi.org/10.1007/s00595-016-1340-6.

GAGNIÈRE, Johan et al. Is Hepatectomy Justified for BRAF Mutant Colorectal Liver Metastases? Annals Of Surgery, [S.L.], v. 271, n. 1, p. 147-154, jan. 2020. Ovid Technologies

(Wolters Kluwer Health).

http://dx.doi.org/10.1097/sla.0000000000002968

GUERRA, Francesco et al. Outcomes of robotic liver resections for colorectal liver metastases. A multi-institutional analysis of minimally invasive ultrasound-guided 
robotic surgery. Surgical Oncology, [S.L.], v. 28, p. 14-18, mar. 2019. Elsevier BV. http://dx.doi.org/10.1016/j.suronc.2018.10.011.

GURUSAMY, Kurinchi et al. Liver resection surgery versus thermal ablation for colorectal LiVer MetAstases (LAVA): study protocol for a randomised controlled trial. Trials, [S.L.], v. 19, n. 1, p. 1-13, 13 fev. 2018. Springer Science and Business Media LLC. http://dx.doi.org/10.1186/s13063-018-2499-5.

INCA (Instituto Nacional do Cancer). Cancer de Instestino, 17 ago. de 2020. Disponível em: https://www.inca.gov.br/tipos-de-cancer/cancer-de-intestino.

Laurent A, Cherqui D, Lesurtel M, Brunetti F, Tayar C, Fagniez PL. Laparoscopic liver resection for subcapsular hepatocellular carcinoma complicating chronic liver disease. Arch Surg. 2003 Jul;138(7):763-9. PMID: 12860758

LEE, Sang et al. Laparoscopic Procedures for Colon and Rectal Cancer Surgery. Clinics In Colon And Rectal Surgery, [S.L.], v. 22, n. 04, p. 218-224, nov. 2009. Georg Thieme Verlag KG. http://dx.doi.org/10.1055/s-0029-1242461.

LUPINACCI, Renato Micelli et al . Manejo atual das metástases hepáticas de câncer colorretal: recomendações do Clube do Fígado de São Paulo. Rev. Col. Bras. Cir., Rio de Janeiro , v. 40, n. 3, p. 251-260, Jun 2013. Disponível https://doi.org/10.1590/S0100-69912013000300016.

Montalti R, Berardi G, Patriti A, Vivarelli M, Troisi RI. Outcomes of robotic vslaparoscopic hepatectomy: A systematic review and meta-analysis. World Journal of Gastroenterology. 2015 Jul. 21(27):8441-8451. http://dx.doi:10.3748/wjg.v21.i27.8441

NACHMANY, Ido et al. Resection of colorectal liver metastases in the elderly-ls it justified? Journal Of Surgical Oncology, [S.L.], v. 113, n. 5, p. 485-488, 11 fev. 2016. Wiley. http://dx.doi.org/10.1002/jso.24188. 
PESI, Benedetta et al. Surgical and oncological outcomes after ultrasound-guided robotic liver resections for malignant tumor. Analysis of a prospective database. The International Journal Of Medical Robotics And Computer Assisted Surgery, [S.L.], v. 15, n. 4, p. 1-6, 27 maio 2019. Wiley. http://dx.doi.org/10.1002/rcs.2002.

POEL, M. J. van Der et al. Laparoscopic combined resection of liver metastases and colorectal cancer: a multicenter, case-matched study using propensity scores. Surgical Endoscopy, [S.L.], v. 33, n. 4, p. 1124-1130, 1 ago. 2018. Springer Science and Business Media LLC. http://dx.doi.org/10.1007/s00464-018-6371-1.

SCUDAMORE, Charles H; BUCZKOWSKI, Andrzej K; SHAYAN, Hossein; HO, Stephen G.F; LEGIEHN, Gerald M; CHUNG, Stephen W; A OWEN, David. Mesohepatectomy. The American Journal Of Surgery, [S.L.], v. 179, n. 5, p. 356360, maio 2000. Elsevier BV. http://dx.doi.org/10.1016/s0002-9610(00)00374-3.

SHE, Wong Hoi; CHEUNG, Tan To; MA, Ka Wing; TSANG, Simon H. Y.; DAl, Wing Chiu; CHAN, Albert C. Y.; LO, Chung Mau. Anatomical Versus Nonanatomical Resection for Colorectal Liver Metastasis. World Journal Of Surgery, [S.L.], v. 44, n. 8, p. 2743-2751, 14 jun. 2020. Springer Science and Business Media LLC. http://dx.doi.org/10.1007/s00268-020-05506-1

UBINK, Inge et al. Surgical and Oncologic Outcomes After Major Liver Surgery and Extended Hemihepatectomy for Colorectal Liver Metastases. Clinical Colorectal Cancer, [S.L.], v. 15, n. 4, p. 193-198, dez. 2016. Elsevier BV. http://dx.doi.org/10.1016/j.clcc.2016.04.006.

VOLPATO, Marília Granzotto; KOCH, Kaiser de Souza. Fatores de mau prognóstico nas peças operatórias de pacientes submetidos ao tratamento cirúrgico do câncer colorretal. Revista Brasileira de Coloproctologia, [S.L.], v. 28, n. 4, p. 414-424, dez. 2008. FapUNIFESP (SciELO). http://dx.doi.org/10.1590/s0101-98802008000400003.

YOO, S.H., ROH, J., CHOI, S. et al. Incidence and risk factors for morbidity and mortality in elderly head and neck cancer patients undergoing major oncological 
surgery.

Cancer

Res

Clin

Oncol 142, 1343-1351

(2016).

https://doi.org/10.1007/s00432-016-2141-4

Enviado: Setembro, 2020.

Aprovado: Outubro, 2020. 